\section{Soft tissue injuries are still a challenge in musculoskeletal sport and exercise medicine}

\author{
Nikos Malliaropoulos, ${ }^{1}$ Mourad Ghrairi, ${ }^{2}$ Yacine Zerguini, ${ }^{3}$ \\ Nat Padhiar ${ }^{4}$
}

Soft tissue injuries in sports are a challenge both for sports and exercise medicine practitioners, athletes, coaches and the team. In this issue we present the latest research on hamstring, ankle, groin and Achilles tendon injuries.

Lateral ankle sprain has a high prevalence both in the athletic and the general population. Even though it is a common injury it can be associated with long term complications such as chronic ankle instability. The Executive Committee of the International Ankle Consortium presents their position recommendations for information, implementation and continued research in order to improve prevention and early management of lateral ankle sprain and reduce the prevalence of long-term sequelae (see pages 1493 and 1496).

Hamstring injuries are still quite a challenge. This edition includes a a PEDro systematic review update on the conservative management for hamstring injuries. This paper shows that core stability and agility exercises may be useful to reduce re-injury rate (see page 1548). In clinical practice two different types of hamstring injuries can be identified: the high-speed running injuries and the stretching type hamstring injuries. Duhig et al (see page 1536) showed that exposing players to large and rapid increases in high speed

${ }^{1}$ QMUL, Centre Sports and Exercise Medicine, London, UK; ${ }^{2}$ Dubai Sports Council, Medical Services, Alshabab Club, Dubai, United Arab Emirates; ${ }^{3}$ Clinique Chahrazed, Médecine et Traumatologie du Sport, Cheraga, Alger; ${ }^{4}$ Queen Mary University of London, Centre for Sports and Exercise Medicine, London, UK

Correspondence to Nikos Malliaropoulos, QMUL, Centre Sports and Exercise Medicine, London, UK; contact@sportsmed.gr running increased their risk of a highspeed running injury, and reducing the volume of high-speed running every four weeks lowered the risk.

Early mobilisation versus immobilisation after Achilles tendon repair is a topic for debate. Phan et al (see page 1550) present a PEDro synthesis on early weight-bearing and rehabilitation versus immobilisation following surgical Achilles tendon repair. It shows there is no significant difference in the likelihood of, or the time taken to return to play from bracing to casting after Achilles tendon repair, but patient satisfaction was higher for the bracing group.

The clinical examination is a fundamental tool for our clinical practice but is often challenged. The study by Serner et al (see page 1541) shows that the clinical examination appears sufficient to diagnose acute adductor injuries, while MRI can assist in accurately locating acute hip flexor injuries.

\section{7: THE BEST OF EUROPEAN SPORT AND EXERCISE MEDICINE, FIFA AND DUBAI}

The European College of Sports and Exercise Medicine Physicians (ECOSEP) welcomes you to the marvellous Gulf city of Dubai on November 25 and 26, 2017.

This will be the 5th ECOSEP Congress, after Thessaloniki, London, Frankfurt and Barcelona. Our conference is one of ECOSEP's major contributions; it helps to promote sport and exercise medicine in Europe, raises the standards of care through education and brings sport and exercise medicine doctors and other experts in the field together. In addition, ECOSEP runs the annual Sports and
Exercise Medicine Traveling Fellowship through a European Sports and Exercise Medicine Center of Excellence network.

For the first time, ECOSEP is holding a congress outside of Europe-our host is the United Arab Emirates Football Association and the FIFA Medical Centre of Excellence. Dubai is an ideal location for East meets West in sport and exercise medicine. The choice of the city of Dubai is not accidental; we were seeking a city that was attractive to the world, that housed a FIFA medical centre of excellence and could provide an ultramodern venue. You will be impressed with the scale and style of the Mohamed Ben Rashid University of Medicine and Health Sciences. Dubai signifies the global characteristics of sport and exercise medicine and the necessity of cross continent collaboration. Collaboration is something we at ECOSEP are increasingly exploring to invest in and we look both within Europe and outside Europe for suitable sport and exercise medicine organisations.

We are proud to partner with FIFA as it launches its foray into the expanded World Cup format. Contemporary topics related to environment and sport, gender definition, and ethics in football medicine will all be remarkable. ECOSEP aims to bring all the 'best of the best' experts and their research from all around the world to Dubai, to meet and discuss sport and exercise medicine topics for two days. We encourage young sport and exercise medicine clinicians to participate, present their scientific research, and liaise with sport and exercise medicine world experts.

We hope to see you all there!

Competing interests None.

Provenance and peer review Not commissioned; externally peer reviewed.

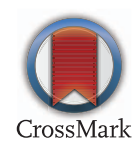

To cite Malliaropoulos N, Ghrairi M, Zerguini Y, et al. Br J Sports Med 2016;50:1487.

Br J Sports Med 2016;50:1487. doi:10.1136/bjsports-2016-097171 